\title{
The Effect of a Surgery Residency Program and Enhanced Educational Activities on Trauma Mortality in Sub-Saharan Africa
}

\author{
Joana Grudziak $^{1} \cdot$ Jared Gallaher $^{1} \cdot$ Leonard Banza $^{2} \cdot$ Bruce Cairns $^{1}$ • \\ Carlos Varela ${ }^{2} \cdot$ Sven Young ${ }^{2,3} \cdot$ Anthony Charles ${ }^{1,2}$
}

Published online: 10 October 2017

\begin{abstract}
Introduction To address the need for more surgical providers in low-resource settings, a collaboration to create a surgical residency-training program for local Malawian physicians was established in 2009. This study sought to describe the short-term independent effect of a surgical residency program on trauma mortality at a tertiary trauma center in sub-Saharan Africa.

Methods We conducted a retrospective analysis of all patients recorded in the trauma surveillance registry of Kamuzu Central Hospital in Lilongwe, Malawi, from 2009 (three residents) through 2014 (11 residents). Logbinominal regression modeling was used to compare the risk ratio of death compared to the referent year of 2009, when the program was started, after adjusting for relevant covariates. Primary injury type was used as a surrogate for injury severity.

Results In total, 82,534 patients were recorded into the KCH Trauma Registry during the study period. Mean age was 23.1 years (SD 15.7) with a male preponderance (72.1\%). Trauma patient volume increased from 8725 patients in 2009 to 15,998 patients in 2014. Each year had a significantly decreased risk of death compared to 2009 when adjusted for primary injury type, age, and gender, with an adjusted risk ratio of 0.73 (95\% CI $0.58,0.90)$ in 2010 and $0.52(95 \%$ CI $0.43,0.62)$ in 2014.

Conclusion The global burden of surgical diseases cannot be attenuated in the presence of an inadequate surgical workforce. After institution of a surgery residency program, adjusted injury-associated mortality decreased each year despite substantial increases in trauma patient volume. In low-resource settings, establishment of a surgical residency program significantly improves trauma-associated outcomes.
\end{abstract}

Electronic supplementary material The online version of this article (doi:10.1007/s00268-017-4272-4) contains supplementary material, which is available to authorized users.

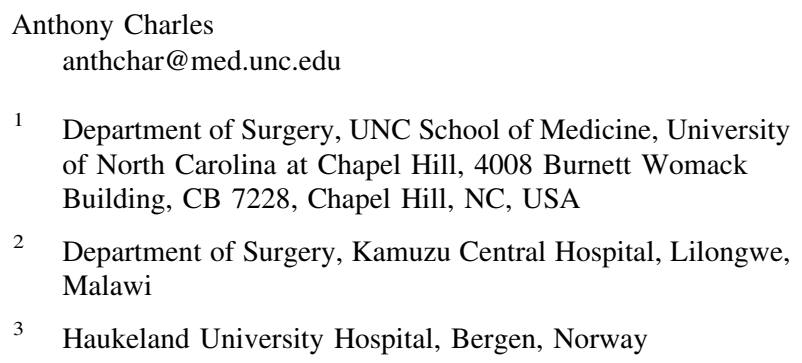

1 Department of Surgery, UNC School of Medicine, University of North Carolina at Chapel Hill, 4008 Burnett Womack Building, CB 7228, Chapel Hill, NC, USA

2 Department of Surgery, Kamuzu Central Hospital, Lilongwe, Malawi

3 Haukeland University Hospital, Bergen, Norway

\section{Introduction}

The significance of traumatic injuries and their impact on global public health is well documented. It is the highest cause of morbidity and mortality in patients younger than 40 years old and the third leading cause of death for all ages, behind only cardiovascular diseases and cancers [1]. In low- and middle-income countries (LMICs), and especially in sub-Saharan Africa, trauma is displacing infectious diseases as a major cause of mortality. There are several reasons why LMICs bear a disproportional burden of trauma morbidity and mortality, such as the absence of a 
trauma system that includes pre-hospital care, management of patients in a trauma center, and the lack of postdischarge rehabilitation. Furthermore, healthcare access issues, due to inadequate government healthcare spending and poor transportation infrastructure, compound this problem. A more pressing concern, however, is a lower overall healthcare workforce, and specifically, a scarcity of surgical workforce.

In sub-Saharan Africa, the availability of all health workers, and especially surgeons, is severely limited in terms of volume and distribution. Africa bears nearly $25 \%$ of the global burden of disease with only $2 \%$ of the global health workforce [2]. Because of economic constraints, most countries in this region have not made the necessary investments in the health sector workforce. Funding priorities that favor infectious diseases have led to the neglect of the development of surgeons, and of the infrastructure necessary to support surgical services. As a result, Africa has less than $1 \%$ of the surgical workforce needed, compared to the USA [3]. The paucity of surgeons in LMICs is also driven by the length of the training required, its cost, and the fact that surgeons can command a higher earning potential in the private sector or abroad, and are hence susceptible to both internal and external "brain drain." The training of surgeons has been further limited due to the reliance of existing healthcare systems on nursing cadres and mid-level providers, or non-physician clinicians, for select surgical procedures.

Malawi, a landlocked country in southeastern Africa, had a per-capita gross domestic product (GDP) of \$350 in 2015 dollars. Its human development index (HDI) ranking of 173 out of 186 countries reflects the challenging health landscape in the country with a life expectancy of only 62 years at birth [4]. The country's only medical school graduates 50 doctors per year, and until 2015, the surgical workforce density in Malawi was only 0.1/100,000 [5].

The Departments of Surgery at Kamuzu Central Hospital $(\mathrm{KCH})$ in Lilongwe, Malawi, and the University of North Carolina at Chapel Hill developed a collaboration in 2006 with visiting surgeons and established a trauma surveillance registry in 2008. $\mathrm{KCH}$ had a functional department of surgery that delivered surgical care with consultant surgeons and clinical officers. A needs assessment of the department was performed, and the need for a surgical training program was identified. A general surgery and orthopedic residency were inaugurated in 2009 in collaboration with Haukeland University in Norway and the Malawi Ministry of Health. KCH is a public, 900-bed tertiary care hospital and serves as a referral center for approximately 6 million people in the central region of Malawi. During the study period, it had a surgical stepdown unit and a four-bed intensive care unit (ICU) with four ventilators. Trauma, emergency general surgery, and orthopedic surgical services are available 7 days a week. General surgery consultants and Malawian general surgery registrars staff the trauma service. Dedicated neurosurgical expertise or subspecialists are not available.

Given this context, we sought to evaluate the effect of the presence of a general surgery-training program on trauma mortality at a tertiary trauma center in Malawi.

\section{Methods}

This study is a retrospective analysis of secondary data from the $\mathrm{KCH}$ Trauma Registry. This registry contains demographic information, clinical characteristics, and outcome data of all patients presenting to the emergency department with traumatic injuries. All patients entered into the database over 6 years, 2009-2014, were included in this study.

\section{Intervention}

In 2008, $\mathrm{KCH}$ applied for the accreditation of a general surgery-training program from the College of Surgeons for East, Central, and Southern Africa and as part of the accreditation requirement demanded a robust educational curriculum, which had previously not existed within the department of surgery. The $\mathrm{KCH}$, UNC, and Haukeland University faculty got together and discuss the best way to deliver the curriculum.

Didactics were delivered weekly with formal lectures over a 2-year period and repeated, to cover the basic principle of surgery including but not limited to the following: surgical techniques and technology, surgical sepsis and its prevention, general principles of management of trauma and surgical critical care; preoperative, intraoperative, and postoperative management; principles of surgical oncology; hematopoietic and lympho-reticular system, pediatric surgery, breast surgery, endocrine surgery, head and neck surgery; thoracic surgery, vascular surgery, gastrointestinal surgery, urology, orthopedics, and neurosurgery. This was supplemented by giving all our residents access to the Surgical Council on Resident Education (SCORE) Curriculum and access to the University of North Carolina Health Sciences Library. See Appendix 1 of Electronic Supplementary Material for more details of the curriculum.

Morning teaching conferences were instituted every weekday with resident discussing cases that presented via the emergency department, or interesting or difficult cases. This was attending run and led and resident were held accountable. Discussions of relevant pathology, patient management, appropriate laboratory and radiological investigations and surgical options and decision making 
occurred. At this morning conferences, residents, medical students, clinical officers, nurses, and nursing students were present. Furthermore, a weekly mortality and morbidity conference was held. This is where deaths and complications from each of the surgical teams were presented and surgical or system-related errors were addressed for opportunities for improvement.

Ward rounds were formalized. Prior to the program, patients were rounded on only twice a week. With the program, residents rounded daily with consultant-led rounds three times per week. This resulted in an improved bedside teaching and early identification of complications. Surgical skills were taught intraoperatively as residents with attendings present covered all cases. Furthermore, all residents were sent to a Basic Surgical Skills course and International Trauma Life Support course within 6 months of starting residency.

The primary aim of this study was to define the relationship between the increase in the volume and experience of surgical residents, and trauma-associated mortality. The primary outcome was crude mortality among all trauma patients who presented to the $\mathrm{KCH}$ emergency department, including those admitted to the hospital. The exposure was defined as the year the patient was evaluated in the emergency department. In 2009, there were five residents, which increased to 13 residents by 2014. Surgical staff otherwise remained stable during this period. Given the strong association between mortality and primary injury type, and in the absence of reliable injury severity scoring, primary injury type was used as a surrogate for injury severity.

We initially examined the baseline characteristics of the study population, comparing those patients across the years of the study. To identify potential confounders of the relationship between evaluation year and death, we examined the distribution of independent variables, comparing means between groups for the continuous variables, or percentages for the categorical variables. Bivariate analysis for the relationship between each of the independent variables and the outcome was performed using Pearson's correlation for the categorical variables and twosample $t$ tests for continuous variables. Medians of nonnormally distributed continuous variables were tested using a Kruskal-Wallis test. When comparing categorical variables with more than two categories, the aggregate of the remaining categories was used as the referent for comparison. Means are reported with standard deviations $( \pm)$ and medians with interquartile ranges (IQR). Overall crude mortality was calculated using any deaths declared in the emergency department and any deaths in the hospital, against all patients recorded in the trauma registry.

We used a log-binomial regression to fit a model estimating the risk ratio for trauma-associated mortality during the years of the study, adjusted for confounders. The first year of the residency, 2009, was used as the referent year. After fitting the initial model with mortality and all potential confounders, we used a change-in-effect method to remove covariates that did not substantially alter the relationship between the year of evaluation and mortality. An adjusted risk ratio and $95 \%$ confidence interval are reported from the estimates of the final model.

All statistical analysis was performed using Stata/SE 13.1 (Statacorp LP, College Station, TX). The Malawi National Health Services Review Committee and the University of North Carolina Institutional Review Board approved this study. The University of North Carolina Department of Surgery and the North Carolina Jaycee Burn Center provided funding.

\section{Results}

During the study period, 82,534 patients were recorded into the KCH Trauma Registry. Yearly trauma patient volume increased from 8725 patients in 2009 to 15,998 patients in 2014. The distribution of age and sex was similar for each year, with a mean age of $23 \pm 15.6$ years, and the female proportion at approximately $28 \%$ (Table 1 ). The proportion of injuries that occurred at home increased from $35.9 \%$ in 2009 to $45.0 \%$ in $2014(p<0.001)$, while other settings remained comparable. Injuries occurred by similar mechanisms throughout the study, with the exception of falls, which increased from $22.5 \%$ in 2009 to $29.4 \%$ in 2014 $(p<0.001)$, and assaults, which decreased from 27.5 to $23.6 \%(p<0.001)$. In all years, most patients were transported to the hospital via minibus (small vans that act as inexpensive taxis) or via private vehicle. However, the use of minibus doubled from $23.3 \%$ in 2009 to $48.3 \%$ in 2014 $(p<0.001)$, while the use of private vehicles declined correspondingly, from 47.9 to $31.3 \%$, respectively $(p<0.001)$. Ambulance utilization remained low in all years, at approximately $12 \%$.

On presentation, at least $95 \%$ of patients either responded to voice or were alert on the Alert, Voice, Pain, Unresponsive Scale (AVPU), which records a patient's level of consciousness as either alert, responding to verbal stimuli, responding to pain stimuli, or unresponsive. The proportion of patients, who presented as unresponsive, while remaining small, more than doubled from $0.6 \%$ in 2009 to $1.3 \%$ in 2013 and $2014(p<0.001)$. Similarly, head injuries, although representing a small proportion of overall injuries, nearly doubled from $2.5 \%$ in 2009 to $4.7 \%$ in $2014(p<0.001)$. The primary injury type throughout the study remained soft tissue injury, ranging from $59.4 \%$ in 2014 to $68.8 \%$ in $2012(p<0.001)$.

Approximately $80 \%$ of all patients were treated and discharged from the emergency department every year 
Table 1 Bivariate analysis of background and injury details by year of evaluation. Patients discharged from the emergency department and those dead on arrival were excluded

\begin{tabular}{|c|c|c|c|c|c|c|c|}
\hline & $\begin{array}{l}2009 \\
(n=1617)\end{array}$ & $\begin{array}{l}2010 \\
(n=1905)\end{array}$ & $\begin{array}{l}2011 \\
(n=2900)\end{array}$ & $\begin{array}{l}2012 \\
(n=2929)\end{array}$ & $\begin{array}{l}2013 \\
(n=2736)\end{array}$ & $\begin{array}{l}2014 \\
(n=2545)\end{array}$ & $p$ value \\
\hline Patient age in years: mean (SD) & $26.9(16.5)$ & $27.0(17.3)$ & $25.4(18.1)$ & $24.3(18.7)$ & $25.0(18.5)$ & $25.9(18.6)$ & $<0.001$ \\
\hline Female gender: $N(\%)$ & $410(25.4)$ & $460(24.2)$ & $713(24.6)$ & $810(27.7)$ & $687(25.1)$ & $613(24.1)$ & 0.027 \\
\hline \multicolumn{8}{|l|}{ Setting of injury: $N(\%)$} \\
\hline Home & $466(28.8)$ & $590(31.0)$ & 1119 (38.6) & $1334(45.5)$ & $1159(42.4)$ & 1059 (41.6) & $<0.001$ \\
\hline Work & $82(5.1)$ & $79(4.2)$ & $127(4.4)$ & $117(4.0)$ & $128(4.7)$ & $117(4.6)$ & 0.12 \\
\hline Road/street & $696(43.0)$ & 909 (47.7) & $1339(46.2)$ & $1137(38.8)$ & $1061(38.8)$ & $1074(42.2)$ & $<0.001$ \\
\hline Public space & $55(3.4)$ & $91(4.8)$ & $83(2.9)$ & $100(3.4)$ & $146(5.3)$ & $136(5.3)$ & $<0.001$ \\
\hline Other & $67(4.1)$ & $101(5.3)$ & $192(6.6)$ & $228(7.8)$ & $230(8.4)$ & $149(5.9)$ & $<0.001$ \\
\hline Missing & $251(15.5)$ & $135(7.1)$ & $40(1.4)$ & $13(0.4)$ & $12(0.4)$ & $10(0.4)$ & $<0.001$ \\
\hline \multicolumn{8}{|l|}{ Mechanism of injury: $N(\%)$} \\
\hline Pedestrian hit by vehicle & $222(13.7)$ & $292(15.3)$ & $370(12.8)$ & $292(10.0)$ & $290(10.6)$ & $262(10.3)$ & $<0.001$ \\
\hline $\begin{array}{l}\text { Driver/passenger in vehicle } \\
\text { accident }\end{array}$ & $412(25.5)$ & $509(26.7)$ & $714(24.6)$ & $572(19.5)$ & $601(22.0)$ & $577(22.7)$ & $<0.001$ \\
\hline Fall & $222(13.7)$ & $291(15.3)$ & $652(22.5)$ & $816(27.9)$ & $750(27.4)$ & $562(22.1)$ & $<0.001$ \\
\hline Assault & 387 (23.9) & $432(22.7)$ & $563(19.4)$ & $578(19.7)$ & $488(17.8)$ & $569(22.4)$ & $<0.001$ \\
\hline Collapsed structure & $59(3.7)$ & $103(5.4)$ & $139(4.8)$ & $148(5.1)$ & $149(5.5)$ & $104(4.1)$ & 0.040 \\
\hline Other & $277(17.1)$ & $249(13.1)$ & $454(15.7)$ & $514(17.6)$ & $442(16.2)$ & $460(18.1)$ & $<0.001$ \\
\hline Missing & $38(2.4)$ & $29(1.5)$ & $8(0.3)$ & $9(0.3)$ & $16(0.6)$ & $11(0.4)$ & $<0.001$ \\
\hline \multicolumn{8}{|l|}{ Transport to hospital } \\
\hline Minibus & $117(7.2)$ & $176(9.2)$ & $526(18.1)$ & $604(20.6)$ & $718(26.2)$ & $603(23.7)$ & $<0.001$ \\
\hline Private vehicle & $694(42.9)$ & $704(37.0)$ & $1067(36.8)$ & $973(33.2)$ & $822(30.0)$ & $751(29.5)$ & $<0.001$ \\
\hline Ambulance & $582(36.0)$ & $761(40.0)$ & $1006(34.7)$ & $1046(35.7)$ & $945(34.5)$ & $945(37.1)$ & $<0.001$ \\
\hline Walked & $19(1.2)$ & $17(0.9)$ & $39(1.3)$ & $47(1.6)$ & $20(0.7)$ & $31(1.2)$ & 0.049 \\
\hline Police & $122(7.5)$ & $141(7.4)$ & $162(5.6)$ & $169(5.8)$ & $137(5.0)$ & $132(5.2)$ & $<0.001$ \\
\hline Other & $50(30.1)$ & $70(3.7)$ & $87(3.0)$ & $86(2.9)$ & $90(3.3)$ & $73(2.9)$ & 0.6 \\
\hline Missing & $22(2.0)$ & $36(1.9)$ & $13(0.5)$ & $4(0.1)$ & $4(0.2)$ & $10(0.4)$ & $<0.001$ \\
\hline \multicolumn{8}{|l|}{ Initial AVPU score: $N(\%)$} \\
\hline Unresponsive & $50(3.1)$ & $61(3.2)$ & $82(2.8)$ & $33(1.1)$ & $32(1.2)$ & $39(1.5)$ & $<0.001$ \\
\hline Responds to pain & $31(1.9)$ & $26(1.4)$ & $27(0.9)$ & $27(0.9)$ & $28(1.0)$ & $30(1.2)$ & 0.22 \\
\hline Responds to voice or alert & $1463(90.5)$ & $1768(92.8)$ & $2659(91.7)$ & $2864(97.8)$ & $2676(97.8)$ & $2473(97.2)$ & $<0.001$ \\
\hline Missing & $73(4.5)$ & $50(2.6)$ & $132(4.6)$ & $5(0.2)$ & $0(0.0)$ & $3(0.1)$ & $<0.001$ \\
\hline \multicolumn{8}{|l|}{ Primary injury type: $N(\%)$} \\
\hline Soft tissue injury & $619(38.3)$ & $682(35.8)$ & 917 (31.6) & $870(29.7)$ & $623(22.8)$ & $506(19.9)$ & $<0.001$ \\
\hline Fracture & $405(25.1)$ & $568(29.8)$ & $1041(35.9)$ & $1063(36.3)$ & $1101(40.2)$ & 957 (37.6) & $<0.001$ \\
\hline Dislocation & $31(1.9)$ & $28(1.5)$ & $68(2.3)$ & $69(2.4)$ & $78(2.9)$ & $56(2.2)$ & 0.07 \\
\hline Head injury & $153(9.5)$ & $250(13.1)$ & $321(11.1)$ & $344(11.7)$ & 407 (14.9) & $443(17.4)$ & $<0.001$ \\
\hline Penetrating wound & $70(4.3)$ & $72(3.8)$ & $103(3.6)$ & $84(2.9)$ & $83(3.0)$ & $117(4.6)$ & 0.003 \\
\hline Burn & $149(9.2)$ & $115(6.0)$ & $257(8.9)$ & $267(9.1)$ & $245(9.0)$ & $268(10.5)$ & $<0.001$ \\
\hline Other & $138(8.5)$ & $153(8.0)$ & $182(6.3)$ & $229(7.8)$ & $195(7.1)$ & $196(7.7)$ & 0.031 \\
\hline Missing & $52(3.2)$ & 37 (1.9) & $11(0.4)$ & $3(0.1)$ & $4(0.2)$ & $2(0.1)$ & $<0.001$ \\
\hline
\end{tabular}

during the study. The proportion of patients admitted to the hospital ward also remained similar, ranging from $15.1 \%$ in 2010 to $19.3 \%$ in $2011(p<0.001)$, but the crude volume increased substantially each year (Fig. 1). Less than $1 \%$ of patients were admitted to the ICU.
Crude mortality for all presenting trauma patients significantly decreased from $2.4 \%$ in 2009 to $1.8 \%$ in 2014 $(p<0.001)$ (Table 2). There were also significant decreases in injury-specific crude mortality for patients with fractures and head injuries. Fracture-related mortality 
decreased from $2.8 \%$ in 2009 to $1.0 \%$ in $2014(p<0.001)$. Head injury-related mortality fell dramatically, from $58.5 \%$ in 2009 to $18.3 \%$ in 2014. Crude mortality due to burn injury increased during the study period, from $2.0 \%$ in 2009 to $6.1 \%$ in 2014. Soft tissue injuries contributed $<1 \%$ to mortality, and mortality from other injury types remained stable each year.

Upon log-binomial modeling, the adjusted risk ratio of death decreased each year compared to 2009, when adjusted for primary injury type, age, and sex. In 2010, the adjusted risk ratio of death was 0.73 (95\% CI $0.58,0.90)$ and fell to 0.52 (95\% CI 0.43, 0.62) by 2014 (Table 2).

A subset analysis to determine the effect of an increased resident complement on emergency department deaths showed that there was no statistically significant difference in ED deaths over the years.

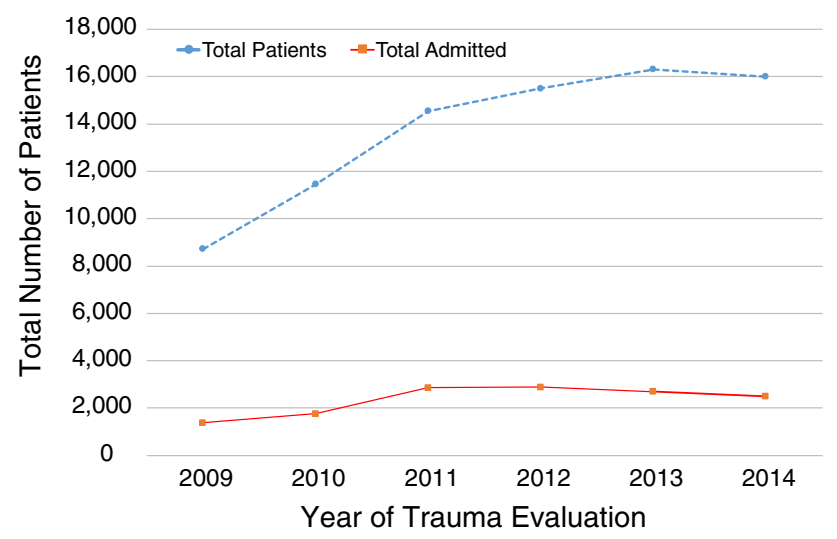

Fig. 1 Total number of trauma patients evaluated and number of patients admitted from 2009-2014

\section{Discussion}

In this study, we show that the presence of a surgical training program significantly reduces the relative risk for trauma mortality by increasing the number of resident surgical providers and improving their education. Along with an increase in surgical trainees during this time, their surgical education, accountability, and adherence to the principles of Advanced Trauma Life Support (ATLS) as set forth by the American College of Surgeons, were reinforced. The reduction in relative risk of mortality from trauma occurred despite the dramatic increase in the number of trauma admissions during the study period. The existing hospital infrastructure, nursing staffing, operating room access, number of anesthesia providers, and adjunctive diagnostic capabilities at $\mathrm{KCH}$ remained the same. More importantly, the number of fully trained surgical faculty, both local and visiting surgeons, was also unchanged.

According to the Lancet Commission on Global Surgery, the global burden of disease amenable to surgical intervention, such as trauma, cancer, and complications from childbirth is substantial and growing. Despite this, there are currently gross disparities in access to safe surgical care worldwide, with an insufficient surgical workforce being a major cause of these inequalities [6, 7]. Surgical care, which has been shown to be cost-effective, could avert $20-40 \%$ of the mortality burden in LMICs, saving nearly 2 million lives annually [8-10]. Despite the magnitude of these estimates, surgery remains a neglected aspect of health systems development in LMICs [11]. Although a critical shortage of surgical providers has been described in many countries, the global number and distribution remain poorly assessed [12]. Many parts of subSaharan Africa average one surgeon for every 250,000

Table 2 Comparison of injury-specific crude mortality by trauma year, excluding those discharged from the emergency department and those dead on arrival. Risk ratios adjusted for injury type, age, and sex

\begin{tabular}{|c|c|c|c|c|c|c|c|}
\hline & 2009 & 2010 & 2011 & 2012 & 2013 & 2014 & $p$ value \\
\hline \multicolumn{8}{|l|}{ Primary injury type morality: $(\%)$} \\
\hline Soft tissue & 7.0 & 2.7 & 2.6 & 2.0 & 1.0 & 2.4 & 0.001 \\
\hline Fracture & 8.6 & 6.4 & 3.5 & 1.5 & 1.1 & 1.7 & $<0.001$ \\
\hline Burn & 33.3 & 16.7 & 16.5 & 17.5 & 14.2 & 13.1 & 0.6 \\
\hline Penetrating wound & 10.0 & 0.0 & 3.9 & 3.6 & 7.2 & 5.1 & 0.5 \\
\hline Head injury & 40.4 & 27.9 & 18.1 & 19.3 & 13.2 & 11.3 & $<0.001$ \\
\hline Other & 6.1 & 0.0 & 8.1 & 3.4 & 3.4 & 2.4 & 0.027 \\
\hline Total deaths: $N$ & 54 & 42 & 134 & 153 & 120 & 125 & $<0.001$ \\
\hline Crude mortality (\%) & 12.6 & 7.8 & 6.2 & 5.4 & 4.5 & 4.9 & $<0.001$ \\
\hline $\begin{array}{l}\text { Adjusted risk ratio of death for all injuries: RR } \\
(95 \% \mathrm{CI})\end{array}$ & 1.00 & $\begin{array}{l}0.64(0.44 \\
0.95)\end{array}$ & $\begin{array}{l}0.56(0.42 \\
0.76)\end{array}$ & $\begin{array}{l}0.49(0.36 \\
0.66)\end{array}$ & $\begin{array}{l}0.36(0.27 \\
0.49)\end{array}$ & $\begin{array}{l}0.35(0.26 \\
0.48)\end{array}$ & \\
\hline
\end{tabular}


people, with some rural areas having one surgeon for every 2.5 million people [13]. As we show here, the training of surgeons and surgical care providers represents both a costand life-saving intervention to address the burden of surgical disease in sub-Saharan Africa.

We have previously demonstrated that the creation of a general surgery-training program in a resource-poor setting increases case volume and case complexity, which is a proxy for an increased surgical access [14]. This study underscores the importance of the presence of a surgical residency program in these settings. As shown previously in the USA, Level I centers with surgical residents have improved patient survival $[15,16]$. So far, a similar demonstration in developing countries has not been reported. Interestingly, there was no difference in ED deaths over the year of study. This was not entirely surprising as ED deaths in our setting can be attributable more to the absence of pre-hospital care and delayed presentations.

The healthcare system in Malawi has universally be run by clinical officers, and indeed, prior to the creation of the residency program, there were over approximately five clinical officers in the department. When we delivered the didactic curriculum to our residents, we made it a point to include all clinical officers and nurses in all aspects, lectures, morning report, and bedside teaching. This concerted effort to deliver surgical education helped all surgical providers-clinical officers and nurses. Regarding trauma care, the UNC faculty leading the effort is a trauma surgeon. Given the burden of trauma in Malawi, trauma education and the management of the critically injured patient were overemphasized. We recognize that not all hospitals have the resources or the capacity to develop a residency program, but our study re-emphasizes the importance of an educational program directed toward available healthcare providers (medical officers, clinical officers, and nurses) to improve their knowledge and skills is imperative if we are to change surgical mortality.

Our study has several strengths. The number of patients recorded in our database is large; the $\mathrm{KCH}$ trauma registry is one of the largest trauma registries in sub-Saharan Africa. The stable complement of other surgical providers and clinicians in the hospital during the study period minimized confounding. Finally, the political stability of Malawi, relatively rare in sub-Saharan Africa, means that the case mix of admissions remained consistent between 2009 and 2014, with no armed conflict.

This study is limited by its retrospective methodology. In addition, traditional injury severity scoring methods, such as the Injury Severity Score or the Revised Trauma Score, were not consistently available through the duration of the study. However, using the primary injury type as a surrogate for injury severity, although not as precise as validated injury severity scores, has internal validity, since the most severe injury has a strong association with mortality in our population. Because we applied the same method for the entire duration of the study, any shortcomings are consistent throughout the analysis.

To our knowledge, this is the first study on the effect of a surgical residency and enhanced educational activities on in-hospital trauma mortality. Though we have not proved causality, we have shown an association. We believe that the effect seen goes beyond merely increasing manpower. Both the quantity and quality of healthcare personnel are important in improving surgical care delivery and outcomes. It is important to emphasize the particularly strong surgical education model that exists at $\mathrm{KCH}$. We believe that both the increase in trainee surgical providers and this commitment to surgical education are critical for the reduction in mortality, and may not be replicated in another setting in the absence of a robust teaching curriculum. In addition, countries that have a surgical workforce density that is closer to the WHO-recommended level might not experience a similar reduction in trauma mortality with the institution of a general surgery program. Indeed, there is evidence that gains from an increased surgical workforce plateau at approximately 40 per 100,000 population, so it is possible that our study does not apply in areas which have already reached this threshold [7].

\section{Conclusion}

We have shown that the development of a new general surgery-training program with the improved educational environment that is created for all healthcare providers is associated with a significant decrease in trauma mortality at a tertiary hospital in a resource-poor setting. This success is attributable to a strategic vision and sustainable collaboration between two foreign institutions, and the Malawi Ministry of Health, which extends beyond the historical mission trip approach of other well-intentioned collaboration. The global burden of surgical diseases cannot be attenuated in the presence of an inadequate surgical workforce. More studies are needed to evaluate the effects of other sustainable programs that focus on surgical workforce on patient access and outcomes in sub-Saharan Africa.

\section{References}

1. Global Burden of Diseases Comparison Tool. http://vizhub. healthdata.org/gbd-compare. Accessed 13 Sept 2016

2. Ozgediz D, Riviello R (2008) The 'other' neglected diseases in global public health: surgical conditions in sub-Saharan Africa. PLoS Med 5:6 
3. McQueen KA, Ozgediz D, Riviello R et al (2010) Essential surgery: integral to the right to health. Health Hum Rights 12(1):137-153

4. World Bank. Country profiles: Malawi. http://data.worldbank. org/country/Malawi. Accessed 2 Sept 2016

5. Henry JA, Frenkel E, Borgstein E et al (2015) Surgical and anaesthetic capacity of hospitals in Malawi: key insights. Health Policy Plan 8:985-994

6. Davies JI, Meara JG (2015) Global surgery-going beyond the Lancet Commission. Lancet 386(9993):507-509

7. Meara JG, Leather AJM, Hagander L et al (2015) Global surgery 2030: evidence and solutions for achieving health, welfare, and economic development. Lancet 386(9993):8-14

8. Chao TE, Sharma K, Mandigo M et al (2014) Cost-effectiveness of surgery and its policy implications for global health: a systematic review and analysis. Lancet Glob Health 2(6):e334-e345

9. Grimes CE, Henry JA, Maraka J et al (2014) Cost effectiveness of surgery in low- and middle-income countries: a systematic review. World J Surg 38(1):252-263. doi:10.1007/s00268-0132243-y

10. Mock C, Joshipura M, Arreola-Risa C et al (2012) An estimate of the number of lives that could be saved through improvements in trauma care globally. World J Surg 36(5):959-963. doi:10.1007/ s00268-012-1553-9

11. Meara JG, Hagender L, Leather AJ (2014) Surgery and global health: a Lancet Commission. Lancet 383(9911):12-13

12. Hoyler M, Finlayson SR, McClain CD et al (2014) Shortage of doctors, shortage of data: a review of the global surgery, obstetrics, and anesthesia workforce. World J Surg 38(1):269-280. doi:10.1007/s00268-013-2324-y

13. Kakande I (2005) The challenge of surgery in Africa. http://www. asecma.org/articulos/Ignatius_Kakande.pdf. Accessed 17 Aug 2016

14. Kendig C, Tyson A, Young S et al (2015) The effect of a new surgery residency program on case volume and case complexity in a sub-Saharan African hospital. J Surg Educ 72(4):e94-e99

15. Garawe T, Cowan LD, Neas B et al (2010) Survival benefit of transfer to tertiary trauma centers for major trauma patients initially presenting to non-tertiary trauma centers. Acad Emerg Med 17(11):1223-1232

16. Cudnik MT, Newgard CD, Sayre MR (2009) Level I versus Level II trauma centers: an outcomes-based assessment. J Trauma 66(5):1321-1326 